\title{
Ultra-triathlon-Pacing, performance trends, the role of nationality, and sex differences in finishers and non-finishers
}

\author{
Sousa, Caio Victor ; Nikolaidis, Pantelis Theodoros ; Knechtle, Beat
}

\begin{abstract}
Ultra-triathlons are defined as triathlons longer than the traditional Ironman distance and became more popular in the last two decades; however, scarce scientific evidence of these events are available. Therefore, we aimed to investigate the trends of performance, pacing, nationality, sex differences, and rate of non-finishers in ultra-triathlons. Data from 1985 to 2018 were collected including Double Iron, Triple Iron, Quintuple Iron, and Deca Iron ultra-triathlons. Different pacing patterns by event and sex were observed $(\mathrm{P}<.05)$; athletes spent less \%time in swimming and cycling, and more \%time in running as the distance of event was longer; women spent more \%time in cycling and less\% time in running in Double and Triple. Performance analysis showed a negative trend over time for men and women since 1985. Switzerland, France, and Germany were the fastest nations in ultra-triathlons. The frequency of North Americans competing in Europe was very low (<5\%), whereas Europeans often competed in North America ( 25\%). The rate of non-finishers between sexes was similar in all races with the exception of Deca Iron ultra-triathlon, which was much greater ( $20 \%)$ for women. Non-finishers had slower race times in swimming and cycling splits than finishers. In conclusion, ultra-triathletes should redistribute their energy among swimming, cycling, and running depending on their sex and distance of race. Performance in ultra-triathlons has been decreasing in men and women over the years, but sex difference in performance remained. Europeans were the fastest ultra-triathletes and compete in Europe and North America. Additionally, non-finishers were slower swimmers and cyclists than finishers.
\end{abstract}

DOI: https://doi.org/10.1111/sms.13598

Posted at the Zurich Open Repository and Archive, University of Zurich

ZORA URL: https://doi.org/10.5167/uzh-178780

Journal Article

Accepted Version

Originally published at:

Sousa, Caio Victor; Nikolaidis, Pantelis Theodoros; Knechtle, Beat (2019). Ultra-triathlon-Pacing, performance trends, the role of nationality, and sex differences in finishers and non-finishers. Scandinavian Journal of Medicine Science in Sports:sms.13598.

DOI: https://doi.org/10.1111/sms.13598 
5

6

7

8

9

\section{Running head: Ultra-triathlon performance analysis}

\author{
Caio Victor Sousa ${ }^{1,2, *}$ \\ Pantelis Theodoros Nikolaidis ${ }^{3}$ \\ Beat Knechtle $e^{4,5}$
}

\section{Correspondence}

Prof. Dr. med. Beat Knechtle

Facharzt FMH für Allgemeinmedizin

Medbase St. Gallen Am Vadianplatz

Vadianstrasse 26

9001 St. Gallen, Switzerland

Telefon / telefax: +41 (0) 712269300 / +41 (0) 712269301

E-mail: beat.knechtle@hispeed.ch 


\section{ABSTRACT}

39 Ultra-triathlons are defined as triathlons longer than the traditional Ironman distance

40 and became more popular in the last two decades; however, scarce scientific evidence

41 of these events are available. Therefore, we aimed to investigate the trends of

42 performance, pacing, nationality, sex differences and rate of non-finishers in ultra-

43 triathlons. Data from 1985-2018 were collected including Double Iron, Triple Iron,

44 Quintuple Iron and Deca Iron ultra-triathlons. Different pacing patterns by event and

45 sex were observed $(p<0.05)$; athletes spent less \%time in swimming and cycling, and

46 more \%time in running as the distance of event was longer; women spent more \%time

47 in cycling and less\% time in running in Double and Triple. Performance analysis

48 showed a negative trend over time for men and women since 1985. Switzerland, France

49 and Germany were the fastest nations in ultra-triathlons. The frequency of North

50 Americans competing in Europe was very low $(<5 \%)$, whereas Europeans often

51 competed in North-America ( 25\%). The rate of non-finishers between sexes was

52 similar in all races with the exception of Deca Iron ultra-triathlon, which was much

53 greater $(\sim 20 \%)$ for women. Non-finishers had slower race times in swimming and

54 cycling splits than finishers. In conclusion, ultra-triathletes should redistribute their

55 energy among swimming, cycling and running depending on their sex and distance of

56 race. Performance in ultra-triathlons has been decreasing in men and women over the

57 years, but sex difference in performance remained. Europeans were the fastest ultra-

58 triathletes and compete in Europe and North America. Additionally, non-finishers were

59 slower swimmers and cyclists than finishers.

60 Keywords: ultra-endurance; swimming; cycling; running; athlete. 


\section{INTRODUCTION}

62

63 The most popular distance in triathlon has been the traditional Ironman format that comprehends $3.8 \mathrm{~km}$ of swimming, $180 \mathrm{~km}$ of cycling and $42 \mathrm{~km}$ of running $\stackrel{1}{ }$. Triathlons with greater distances than Ironman were usually called ultra-triathlons, in that regard, races that comprehended two, three or even ten times the distance of an Ironman has

67 being held since $1985^{2}$. Since the beginning, ultra-triathlons increased their number of men and women participants becoming more popular every year ${ }^{2}$. Ultra-triathlons were one of the most challenge endurance events that an athlete could be part of. Even very good and experienced athletes that undertook this kind of races had a great risk to quit the race (i.e. non-finishers). Research showed that gastrointestinal issues was the main reason for dropouts in an Ironman race $^{3}$, but there were no scientific evidences to support athletes and coaches in their race strategy to an ultra-triathlon to avoid becoming a non-finisher.

Much has changed in exercise training in the past decades due to the advances in sports sciences, which could help coaches and athletes to achieve better performances than 10 or 15 years ago. For instance, the number of women participants has been increased in several sports decreasing the men-to-women ratio ${ }^{4-6}$. Moreover, women seemed to close the performance gap with men in several endurance sports, such as open-water swimming ${ }^{4}$, running ${ }^{5}$ or even Ironman triathlon ${ }^{6}$. But there were no such records in ultra-triathlons; instead, it was shown that the best women were actually increasing the

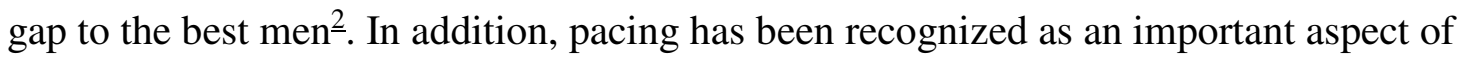
performance in ultra-endurance ${ }^{7, \underline{8}}$; however, no study has compared the relative 
distances. Such information would be of great practical value for ultra-triathletes as they

87 might compete in races of different distances, and likely should re-distribute their energy among the disciplines.

89

90 Furthermore, nationality may play its role through the climatic and cultural differences

91 in each nation which may ease or difficult triathlon practice. The nationality impact in

92 performance and number of participants could be of assistance to event organizers in order to set races in the most preferred locations for each nation, and where may be the fastest athletes. Some previous analysis demonstrated that no nation had a particular dominance in open-water swimming ${ }^{4}$, whereas regarding to running East-Africans (i.e.

96 Ethiopia and Kenya) were the fastest marathoners and ultra-marathoners ${ }^{9}$. Moreover, an 97 analysis of Ironman triathlon demonstrated that nationality played an important role in 98 pacing in all three disciplines and overall race time $\mathrm{in}^{\underline{10}}$.

99

100 However, for the best of our knowledge the role of nationality, finisher/non-finisher, sex

101 and performance trends such as pacing in ultra-triathlon has not yet been investigated.

102 Therefore, the aim of the study was to investigate the trends of performance, pacing, nationality role, sex differences and rate of non-finishers in Double Iron, Triple Iron,

104 Quintuple Iron and Deca Iron ultra-triathlons held from 1985 to 2018. 


\section{Ethics approval}

108 This study was approved by the Institutional Review Board of Kanton St. Gallen,

109 Switzerland, with a waiver of the requirement for informed consent of the participants

110 as the study involved the analysis of publicly available data.

\section{Sample}

113 All data were the official results from Double Iron, Triple Iron, Quintuple Iron and

114 Deca Iron ultra-triathlons held from 1985 to 2018. One of the authors collected all split

115 and overall race times from race websites and organizers. The standard and traditional

116 Ironman distance was $3.8 \mathrm{~km}$ swim / 180km cycle / 42km run, thus the ultra-triathlon

117 distance was as follow: Double Iron (7.6km swim / 360km cycle / 84km run) with 2,988

118 entries/athletes; Triple Iron (11.8km swim / 540km cycle / 126km run) with 1,724

119 entries/athletes; Quintuple Iron (19km swim / 900km cycle / 210km run) with 117

120 entries/athletes; and Deca Iron ultra-triathlon (38km swim / 1800km cycle / 420km run)

121 with 220 entries/athletes; totalizing 5,049 athletes/entries. Competitors needed to

122 perform a distance equal to Ironman distance per day in consecutive days. Data from

123 both women and men were analyzed including overall and split race times, nationality,

124 finisher/non-finisher status, event year and event host nation. Overall and split race

125 times by event and sex were displayed in Table 1.

\section{Data analysis}

128 Continuous data had normality and homogeneity assessed with Kolmogorov-Smirnov's and Levene's test, respectively. Since all continuous variables presented parametric 
130 distribution, data were expressed and mean and standard deviation ( \pm ). Categorial

131 variables were analyzed as frequency rates, Chi square and Cramer's Phi. The

132 percentages of split race times to overall race times were calculated individually for

133 each athlete and the mean and SD displayed in Table 1 (reason why the sum of \% R in

134 each event does not equals $100 \%$ ).

135

136

** Table 1 about here **

137

138 Linear regressions were applied for performance trends. Performance trends with just

139 the top-3 of each race in year were also performed. A one-way ANOVA was applied to

140 compare to race times (overall and splits) between the nations in each event. A student

$141 t$-test for independent samples was applied to compare the speed in splits (i.e.

142 swimming and cycling) between finishers and non-finishers in each event. The

143 significance level was 5\% $(p<0.05)$. All procedures were performed using Statistical

144 Software for the Social Sciences (SPSS v21.0, Chicago, IL, USA) and GraphPad Prism

145 (Prism v6.0, San Diego, CA, USA). 
148 The percentage of spent in each discipline differed among the events $(p<0.001)$. In the 149 swim split, significant differences among all events were identified $(p<0.001)$ with the 150 longer the race, the smaller the percentage (i.e., less relative time). For cycling, Double 151 Iron had significantly larger \% time than Triple and Deca Iron $(p<0.001)$, whereas in

152 the running split, the longer the race higher the percentage, with statistical significant among all comparisons $(p<0.001)$. Additionally, women spent significantly more $\%$ time cycling than men, whereas men spent significantly more \% time running in Double and Triple Iron. No sex differences were identified for Quintuple and Deca Iron.

157 The overall performance trend results revealed an increase in the average race time in 158 all four events from 1985 to 2018 for both men and women, with an exception for the 159 Deca Iron ultra-triathlon in females, which had a negative slope (Figure 1-A to 1-D). 160 The performance trends with only the top-3 race times in each annual race showed a 161 positive slope in overall race time for both women and men in all three events of 162 Double Iron, Triple Iron, Quintuple Iron and Deca Iron ultra-triathlon (Figure 1-E to 1$163 \mathrm{H})$.

** Figure 1 about here **

167 For the performance trends of split race speed in females, negative slopes for the all 168 three disciplines (swim, cycle, run) were identified in Double, Triple and Quintuple events, whereas the Deca Iron ultra-triathlon showed a positive slope (Figure 2-A to 2D). Likewise, performance trends of split race speed in males showed negative slopes 
171 for the all three disciplines (i.e. swim, cycle, and run) in Double, Triple and

172 Quintuple events, whereas Deca Iron ultra-triathlon showed a positive slope for the 173 cycling split but negative for swimming and running (Figure 2-E to 2-H)

174

$175 * *$ Figure 2 about here **

176

177 The comparison of overall race times showed that Switzerland was the fastest in Double 178 Iron $(p<0.001)$, whereas France was the fastest in Triple $(p<0.001)$, Quintuple $(p=$ 179 0.014), and Deca Iron ultra-triathlon $(p<0.001)$. Furthermore, the nation with a higher 180 participation in Double Iron and Quintuple Iron was the United States (USA), in Triple 181 Iron Germany, and Deca Iron ultra-triathlon France (Figure 3).

** Figure 3 about here **

184

185

The comparison of split race times between nations were statistical significant for all three disciplines in Double, Triple and Deca Iron ultra-triathlon, but only in running for

187 the Quintuple Iron (Figure 4). In the Double Iron and Triple Iron, Switzerland had 188 fastest swimmers and cyclists whereas Austria and France had the fastest runners (Figure 4-A and 4-B). In Quintuple and Deca Iron ultra-triathlon, France and Germany were the fastest in all three disciplines, being France the first in cycling and running

191 (Figure 4-C and 4-D).

192 
195 Regarding the participation of Europeans and athletes living in North America (i.e.

196 USA, Canada and Mexico) in events, Europeans presented the majority of participants

197 in races taking place in Europe in all four events (i.e. Double Iron, Triple Iron,

198 Quintuple Iron and Deca Iron ultra-triathlons). Moreover, Europeans also showed a

199 massive participation in races taking place in North America, being more than $25 \%$ in

200 Double and Triple and the majority in Deca Iron ultra-triathlon (Figure 5).

201

202

** Figure 5 about here **

203

204

The rate of participants who did not finish (DNF) a race was similar between women and men in Double Iron ( 7.0\%), Triple Iron ( $5.1 \%)$, and Quintuple Iron $(\sim 8.8 \%)$, but the differences were larger in Deca Iron ultra-triathlon, being of approximately $22 \%$ more DNF in women. Deca Iron ultra-triathlon was the event with less DNF for men, whereas Triple Iron was the event with less DNF for women (Table 2).

Split times (i.e. swimming and cycling) of DNF were higher for both men and women in Double and Triple Iron ultra-triathlon, with an exception for cycling in women in the Triple Iron ultra-triathlon (Figure 6-A and 6-B). DNF men swimmers were slower than

215 finishers in Quintuple Iron ultra-triathlon (Figure 6-C). No statistical significances were 216 identified for Deca Iron ultra-triathlon (Figure 6-D). 
222 The main findings of the present study were that the athletes spent less $\%$ time in swimming and cycling, and more \% time in running as the distance of event was longer; women spent more \% time in cycling and less \% time in running in Double and Triple Iron ultra-triathlon. In addition, the overall race time became slower across calendar years for all events. We hypothesized that this would be a result of increasing number of participants, but even when only including the top three the race time slope remained positive. Furthermore, Switzerland and France were the fastest nations in ultratriathlons, where swimming and cycling were the best disciplines for Switzerland and cycling and running for France. Additionally, North-Americans competed mostly in events held in North-America, whereas Europeans competed in both Europe and North-

232 America. The rate of non-finishers was similar for women and men in all events except the Deca Iron which was higher for women, and non-finishers swim and cycle was slower than in finishers in all events for both men and women.

With the regards to pacing, the differences in the relative contribution of each discipline to the overall race time indicated that triathletes redistributed their energy among the three disciplines depending on the distance of the races. Moreover, the findings suggested that triathletes became relatively faster in swimming and cycling, and relatively slower in running as the distance increased. In turn, this observation might be

241 attributed to an increased exercise-induced fatigue in running compared to the other two

242 exercise modes. It has been previously suggested that exercise economy was also

243 important for performance in addition to maximal oxygen uptake ${ }^{11}$. In events of longer distance, running became more an "intermediate" discipline - a discipline that would be 
245 followed by the discipline of the next day (swimming) - rather than a "last" discipline.

246 Instead, when running was the "last" discipline - as it happened more often in the

247 shorter distances, the triathletes could perform it at a higher exercise intensity (i.e. less

248 economical $)^{\frac{12}{}}$.

249

250 The performance trends results were opposite from what has been observed in open-

251 water swimming $\frac{13}{3}$, marathons ${ }^{14}$ or Ironman triathlon ${ }^{6}, \underline{15}$ showing an improvement in

252 performance over the years. A previous analysis from ultra-triathlons also showed a

253 positive slope for race winners from 1985 to $2009^{2}$. We hypothesized that the reduced

254 mean performance would be a reflection of an increased number of participants, but

255 when analyzing only the top three athletes in each race performance continued in a

256 positive slope for overall race time for both men and women in all four events for

257 Double Iron, Triple Iron, Quintuple Iron and Deca Iron ultra-triathlon. Since there is

258 evidence that elite triathletes were becoming faster every year $\underline{15}, \underline{16}$, it is possible that

259 ultra-triathlons were becoming less popular among the fastest triathletes that may be

260 focusing on different challenges, such as a classification to the Ironman World

261 Championship in Kona-Kailua, Hawaii $\frac{17}{}$, and are now attracting older and experienced

262 athletes not so focused on the race time performance.

264 Regarding the nationality analysis, Europeans were racing in both Europe and North

265 America, whereas athletes living in North America (i.e. Mexico, USA and Canada) have only participated in events taking place in North America. This result may reverberate in performance, since Switzerland, France and Germany were the fastest nations in the present analysis. This is a novelty, since triathlon was originated in the

269 USA $\underline{17, \underline{18}}$ and the World Championship and qualifying races of Ironman have been 270 dominated by women and men from the USA ${ }^{19}$. However, this scenario has been 
271 changing in the last two decades $\frac{10}{}$. Furthermore, some previous analysis of Double and

272 Triple Iron ultra-triathlon also showed a dominance of Europeans in these events $\underline{20}$.

273

274 The rate of non-finishers was similar between men and women in Double Iron, Triple

275 Iron, Quintuple Iron, but much higher in Deca Iron ultra-triathlon with an increased rate

276 of non-finisher women. It was previously described that an ultra-triathlon leads to a loss

277 skeletal muscle mass $\stackrel{21}{ }$. Although women possibly lose less muscle mass than men due

278 to their reduced initial values, it may be enough to cause greater impact in their

279 performance than men $\stackrel{22}{ }$. Thus, body composition may be a limiting factor for the

280 discrepant performance and the ability to finish such a race. On the other hand, it is

281 noteworthy that women have being closing the performance gap with men in several endurance sports including Ironman triathlon ${ }^{\underline{6}}$, but the sex difference does not seem to reduce over time in ultra-triathlons.

284

The average split race times of men and women non-finishers were higher than in finishers. Although a slower pace may save more energy, may be a reflect of less experience and physical fitness to the race, and it also exposes that athlete to an increased time over exercise stress, which means more hydration, food and also a possible gastrointestinal discomfort $\underline{23}^{\underline{24}}$, , a main cause for dropouts in Ironman races ${ }^{3}$. Some previous analysis in pacing of ultra-triathletes showed that best overall athletes were slower in swimming and cycling but fastest in running $\underline{12}$. In sum, ultra-triathletes

292 should account their experience and limitations to set the best individual strategy to which discipline to go faster. 
297 This is an update analysis of ultra-triathlons with all available data from races that have 298 taken place from 1985 to 2018 . The limitations of the present study may be accountable 299 for some unavailable information that could help explain some results, such as the years 300 of experience in triathlons (and/or ultra-triathlons). Furthermore, scientific literature

301 lacks information regarding physiological responses before (preparation), during and

302 after an ultra-triathlon, such information would for a great assistance to understand both 303 great performances and physiological factors for dropouts.

304

305 In conclusion, ultra-triathletes should redistribute their energy among swimming,

306 cycling and running depending on their sex and distance of a race. Also, the average

307 performance in ultra-triathlons decreased in both men and women over the years, but 308 the number of participants increased. Europeans, more specifically Swiss, French and

309 Germans, were the fastest ultra-triathletes, especially Switzerland's swimmers, France's 310 cyclists and Germany's runners when considering performance by discipline. Europeans

311 seemed to compete in races in Europe and North America, whereas North Americans

312 competed mostly in North America. The performance gap between men and women

313 remained unchanged in ultra-triathlons. Additionally, non-finishers were slower

314 swimmers and cyclists than finishers, and women had more dropouts than men in Deca

315 Iron ultra-triathlon. 


\section{REFERENCES}

317 1. Lepers R. Analysis of Hawaii ironman performances in elite triathletes from 3181981 to 2007. Medicine and science in sports and exercise. 2008;40(10):1828-1834.

319 2. Knechtle B, Knechtle P, Lepers R. Participation and performance trends in ultra320 triathlons from 1985 to 2009. Scandinavian journal of medicine \& science in sports. $321 \quad 2011 ; 21(6): e 82-90$.

322 3. Dallam GM, Jonas S, Miller TK. Medical considerations in triathlon 323 competition: recommendations for triathlon organisers, competitors and coaches. Sports 324 medicine. 2005;35(2):143-161.

325 4. Nikolaidis PT, de Sousa CV, Knechtle B. Sex difference in long-distance open326 water swimming races - does nationality play a role? Res Sports Med. 2018;26(3):332327344.

328 5. Nikolaidis PT, Rosemann T, Knechtle B. A Brief Review of Personality in 329 Marathon Runners: The Role of Sex, Age and Performance Level. Sports (Basel). $330 \quad 2018 ; 6(3)$.

331 6. Knechtle B, Kach I, Rosemann T, Nikolaidis PT. The effect of sex, age and 332 performance level on pacing of Ironman triathletes. Res Sports Med. 2019;27(1):99-111. 333 7. Knechtle B, Rosemann T, Lepers R, Rust CA. A comparison of performance of 334 Deca Iron and Triple Deca Iron ultra-triathletes. Springerplus. 2014;3:461.

335 8. Herbst L, Knechtle B, Lopez CL, et al. Pacing Strategy and Change in Body 336 Composition during a Deca Iron Triathlon. Chin J Physiol. 2011;54(4):255-263.

337 9. Nikolaidis PT, Onywera VO, Knechtle B. Running Performance, Nationality, 338 Sex and Age in 10km, Half-marathon, Marathon and 100km Ultra-marathon IAAF 1999-2015. The Journal of Strength \& Conditioning Research. 2016. 
340 10. Nikolaidis PT, Kach I, Rosemann T, Knechtle B. The Role of Nationality on the

341 Pacing of Ironman Triathletes. Asian journal of sports medicine. 2017;8(4).

342 11. O'Toole ML, Douglas PS. Applied physiology of triathlon. Sports medicine. $1995 ; 19(4): 251-267$.

344 12. Knechtle B, Sousa CV, Simões HG, Rosemann T, Nikolaidis PT. Effects of the

345 performance level and race distance on pacing in ultra-triathlons. Journal of human $346 \quad$ kinetics. 2019.

347 13. Nikolaidis PT, Di Gangi S, de Sousa CV, Valeri F, Rosemann T, Knechtle B.

348 Sex difference in open-water swimming-The Triple Crown of Open Water Swimming 349 1875-2017. PloS one. 2018;13(8):e0202003.

350 14. Knechtle B, Di Gangi S, Rust CA, Rosemann T, Nikolaidis PT. Men's

351 Participation and Performance in the Boston Marathon from 1897 to 2017. International 352 journal of sports medicine. 2018.

353 15. Gallmann D, Knechtle B, Rust CA, Rosemann T, Lepers R. Elite triathletes in 354 'Ironman Hawaii' get older but faster. Age. 2014;36(1):407-416.

355 16. Sousa CV, Barbosa LP, Sales MM, et al. Cycling as the best sub-8-hour 356 performance predictor in full distance triathlon. Sports (Basel). 2019.

357 17. Knechtle B, Nikolaidis PT, Rosemann T, Rust CA. [Ironman Triathlon]. Praxis 358 (Bern 1994). 2016;105(13):761-773.

359 18. Sousa CV, Aguiar SS, Olher RR, et al. Hydration status after an Ironman 360 triathlon: a meta-analysis. Journal of Human Kinetics. 2019; Ahead of print.

361 19. Stiefel M, Rust CA, Rosemann T, Knechtle B. A comparison of participation 362 and performance in age-group finishers competing in and qualifying for Ironman 363 Hawaii. International journal of general medicine. 2013;6:67-77. 
364 20. Jeffery S, Knechtle B, Rüst CA, Knechtle P, Rosemann T, Lepers R. European 365 dominance in Triple Iron ultra-triathlons from 1988 to 2011. Journal of Science and 366 Cycling. 2012;1(1):30.

367 21. Knechtle B, Duff B, Amtmann G, Kohler G. An ultratriathlon leads to a 368 decrease of body fat and skeletal muscle mass--the Triple Iron Triathlon Austria 2006.

369 Res Sports Med. 2008;16(2):97-110.

370 22. Sanborn CF, Jankowski CM. Physiologic considerations for women in sport. 371 Clin Sports Med. 1994;13(2):315-327.

372 23. Jeukendrup AE, Jentjens RL, Moseley L. Nutritional considerations in triathlon. 373 Sports medicine. 2005;35(2):163-181.

374 24. Sharwood KA, Collins M, Goedecke JH, Wilson G, Noakes TD. Weight 375 changes, medical complications, and performance during an Ironman triathlon. British 376 journal of sports medicine. 2004;38(6):718-724. 
377 Table 1 Overall and split race time (minutes) of women and men in ultra-triathlons 378 from 1985 to 2018

\begin{tabular}{rcccc}
\hline & Double & Triple & Quintuple & Deca \\
\hline Overall & & & & \\
Female & $1,862.1 \pm 255.6$ & $3,004.8 \pm 440.8$ & $7,338.9 \pm 1,390.3$ & $17,667.3 \pm 1,858.4$ \\
Male & $1,734.0 \pm 250.8$ & $2,875.8 \pm 413.6$ & $6,698.0 \pm 1,210.0$ & $15,983.3 \pm 2,479.6$ \\
Swimming & & & & \\
Female & $172.6 \pm 33.9$ & $255.9 \pm 35.5$ & $564.0 \pm 143.6$ & $1,221.3 \pm 368.6$ \\
$\% \mathrm{R}$ & $9.1 \pm 1.6^{\mathrm{e}}$ & $8.5 \pm 1.3^{\mathrm{e}}$ & $7.7 \pm 1.8^{\mathrm{e}}$ & $6.7 \pm 1.0^{\mathrm{e}}$ \\
Male & $159.9 \pm 33.0$ & $243.9 \pm 43.2$ & $495.5 \pm 87.8$ & $1,088.9 \pm 267.6$ \\
$\% \mathrm{R}$ & $9.1 \pm 1.6^{\mathrm{e}}$ & $8.4 \pm 1.2^{\mathrm{e}}$ & $7.4 \pm 1.3^{\mathrm{e}}$ & $6.7 \pm 1.5^{\mathrm{e}}$ \\
Cycling & & & \\
Female & $947.9 \pm 142.7$ & $1,511.3 \pm 224.9$ & $3,778.3 \pm 841.2$ & $9,129 \pm 2,092.4$ \\
$\% \mathrm{R}$ & $50.1 \pm 3.5^{\mathrm{d}^{*}}$ & $50.1 \pm 3.5^{*}$ & $49.7 \pm 3.4$ & $48.5 \pm 5.0$ \\
Male & $860.3 \pm 136.2$ & $1,412.7 \pm 225.3$ & $3.323 .9 \pm 720.7$ & $7,951.7 \pm 1,603$ \\
$\% \mathrm{R}$ & $49.3 \pm 3.6^{\mathrm{bd}}$ & $48.8 \pm 4.2$ & $48.5 \pm 4.3$ & $48.4 \pm 4.6$ \\
Running & & & & \\
Female & $752.1 \pm 143.7$ & $1,235.5 \pm 257.9$ & $3.068 .8 \pm 575.9$ & $7,794.2 \pm 835.3$ \\
$\% \mathrm{R}$ & $40.2 \pm 3.7^{*}$ & $40.9 \pm 3.9^{\mathrm{d}^{*}}$ & $41.9 \pm 3.6$ & $44.4 \pm 4.7$ \\
Male & $715.9 \pm 139.6$ & $1,225.7 \pm 247.5$ & $2,911.2 \pm 669.6$ & $7,148.6 \pm 1,464.8$ \\
$\% \mathrm{R}$ & $41.1 \pm 4.1^{\mathrm{e}}$ & $42.4 \pm 4.5^{\mathrm{d}}$ & $43.4 \pm 5.4$ & $44.6 \pm 4.9$ \\
\hline
\end{tabular}
males. 
384 Table 2 Number and rate of women and men finishers and non-finishers in ultra385 triathlons from 1985 to 2018

\begin{tabular}{lcccccc}
\hline & \multicolumn{3}{c}{ Female } & \multicolumn{2}{c}{ Male } & $\begin{array}{c}p \text {-value } \\
(\text { Cramer's } \phi)\end{array}$ \\
\hline Double & & F & DNF & F & DNF & \\
& $\mathrm{N}$ & 246 & 89 & 2,133 & 520 & 0.004 \\
& $\%$ & 73.4 & 26.6 & 80.4 & 19.6 & $(0.003)$ \\
Triple & & & & & & \\
& $\mathrm{N}$ & 136 & 33 & 1172 & 383 & 0.156 \\
& $\%$ & 80.5 & 19.5 & 75.4 & 24.6 & $(0.141)$ \\
Quintuple & & & & & \\
& $\mathrm{N}$ & 15 & 6 & 77 & 19 & 0.386 \\
& $\%$ & 71.4 & 28.6 & 80.2 & 19.8 & $(0.374)$ \\
Deca & & & & & \\
& $\mathrm{N}$ & 17 & 10 & 164 & 29 & 0.012 \\
& $\%$ & 63.0 & 37.0 & 85.0 & 15.0 & $(0.005)$ \\
\hline
\end{tabular}

F: finishers; DNF: non-finishers 


\section{FIGURES LEGENDS}

390 Figure 1. Performance trends of overall race time of men and women competing in ultra-triathlons from 1985 to 2018. Panels E, F, G and H included only top-3 athletes in each race.

393

394 Figure 2. Performance trends of split race time of women (panels A, B, C and D) and men (panels E, F, G and H) competing in ultra-triathlons from 1985 to 2018.

396

397 Figure 3. Overall race times (solid black circles) and participation (gray bars) in ultratriathlons of different nations from 1985 to 2018.

399

400 Figure 4. Split race times (swimming, cycling and running) of different nations 401 competing in ultra-triathlons from 1985 to 2018. *: statistical significance, between402 nation effect.

403

404 Figure 5. Frequency rate of participation of Europeans, people born in North America 405 (US, Canada and Mexico) in events that takes place in Europe or North America.

406

407 Figure 6. Split race times of finishers and athletes that do not finished (DNF) ultra408 triathlons. Data from men (blue) and women (red) from 1985 to 2018. *: statistical 409 difference between the groups $(p<0.05)$. 
Figure 1.

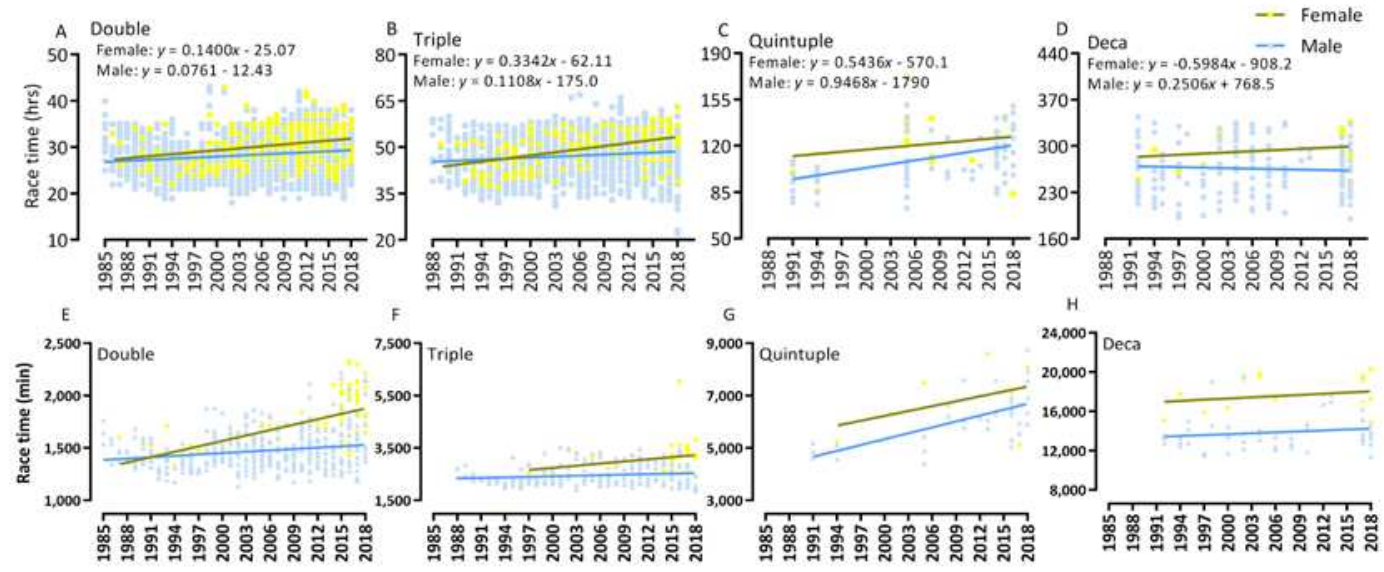



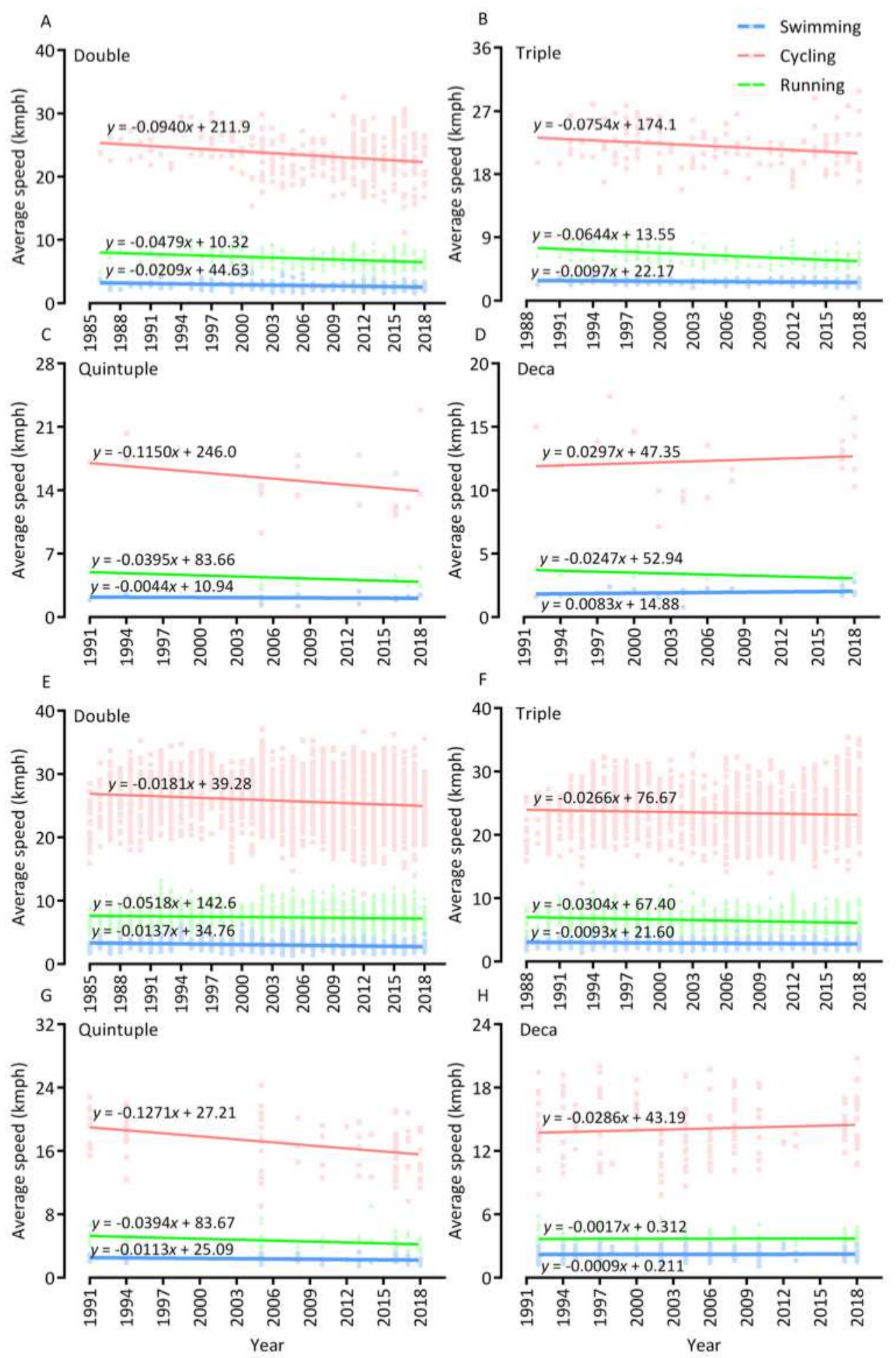
Figure 3.

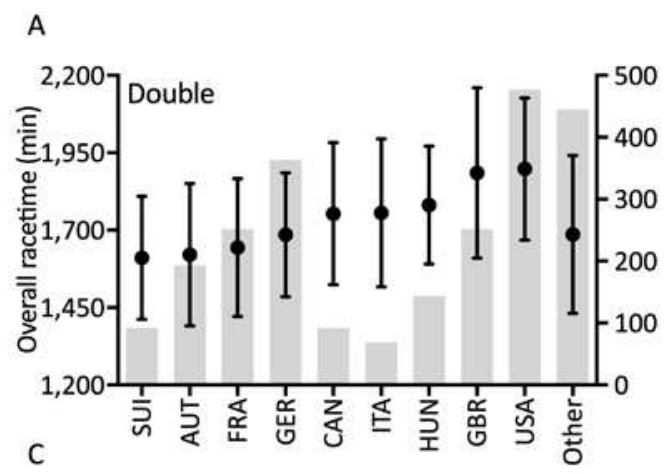

B
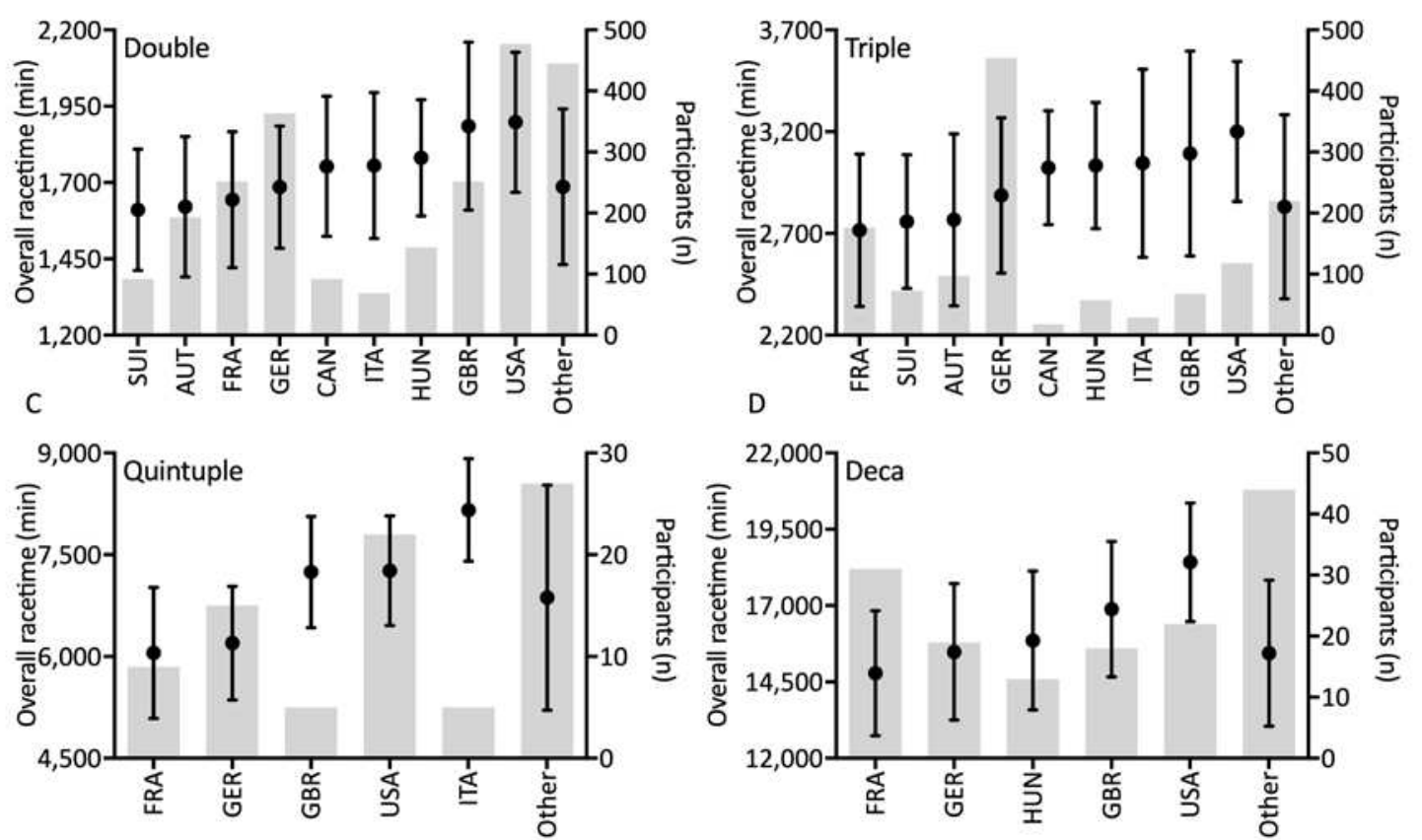

416

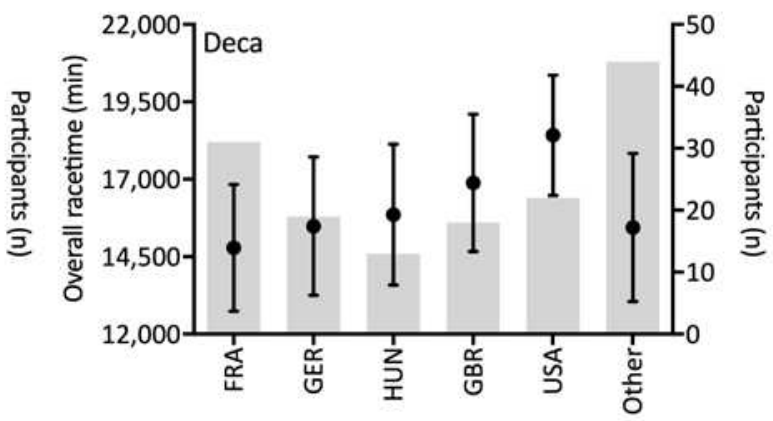


A

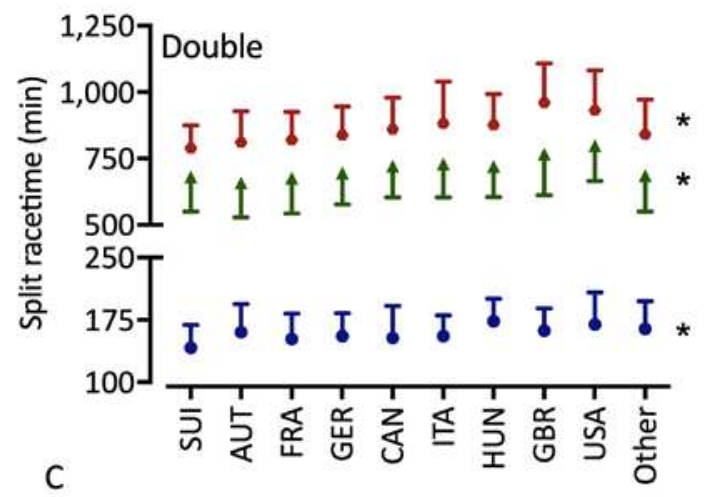

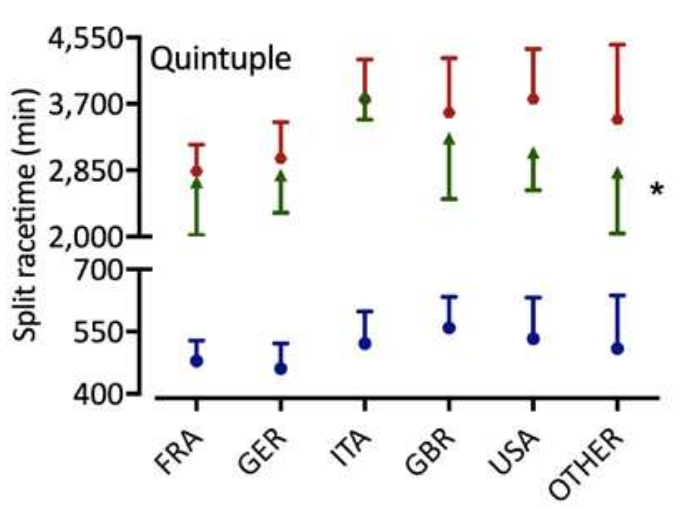

B

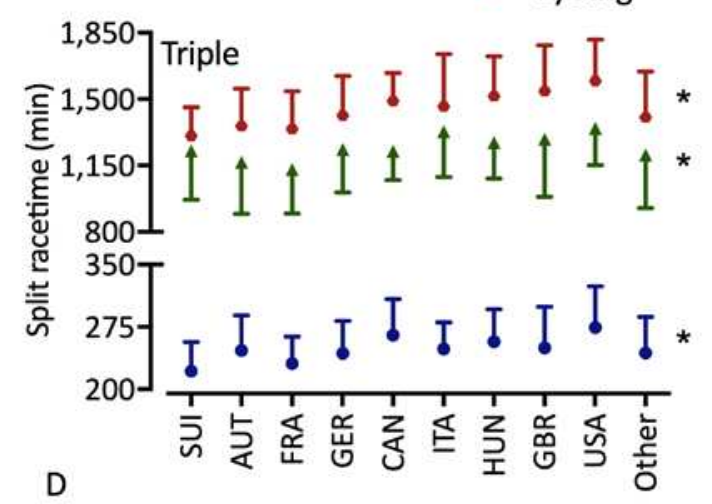

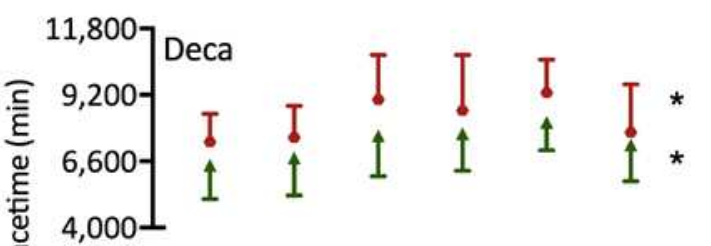

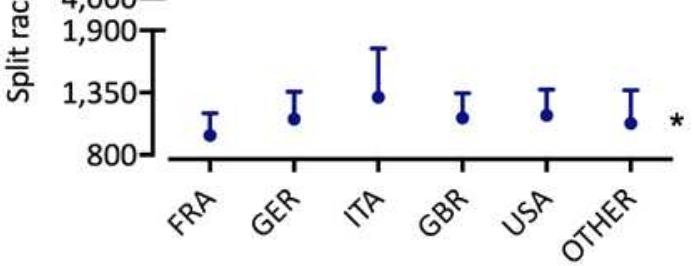

A Running

- Swimming

- Cycling

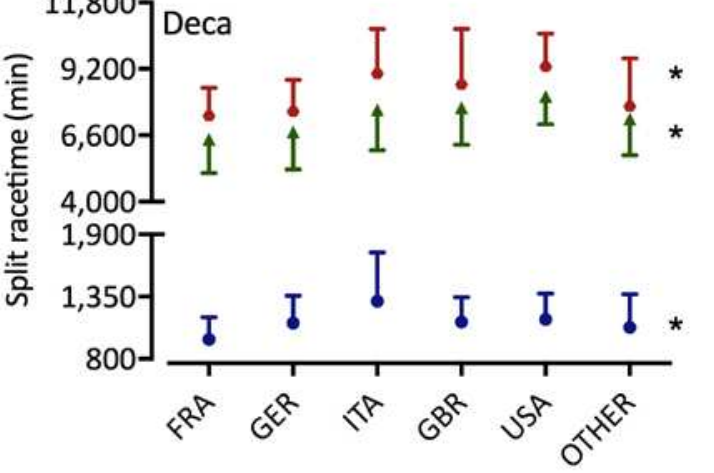


$419 \quad$ Figure 5.

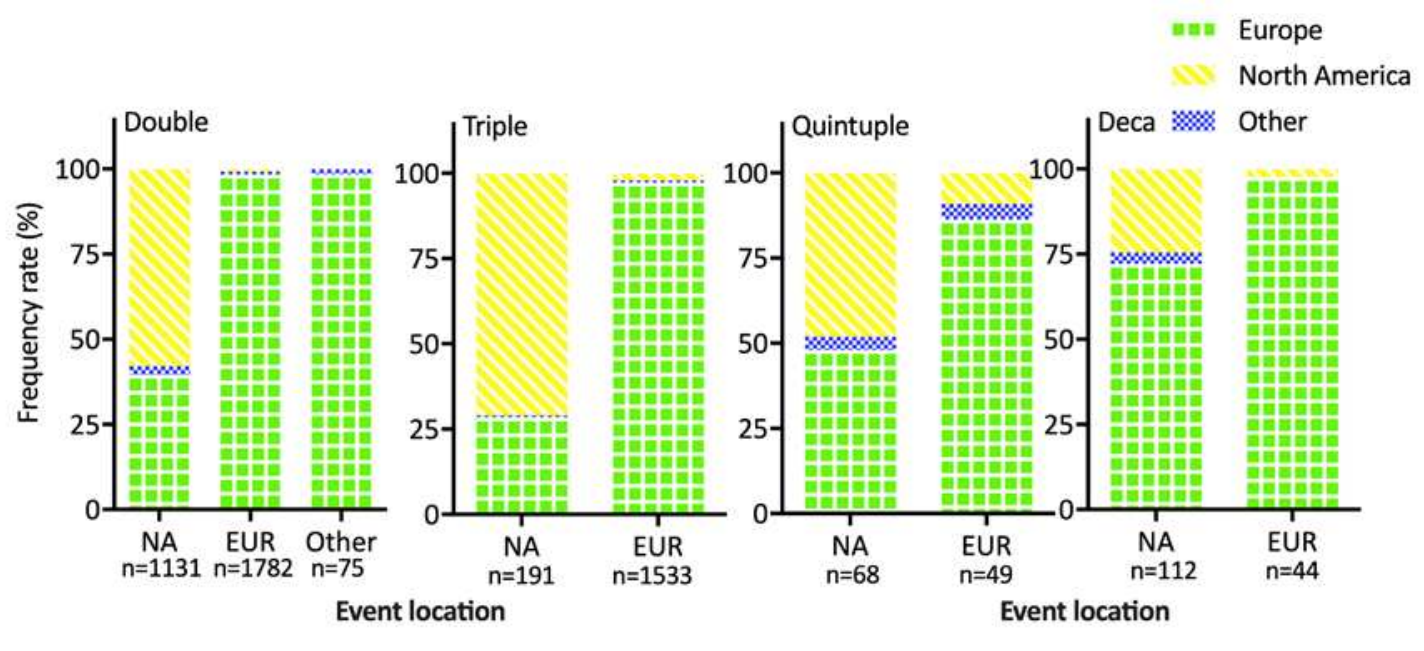

421 
$422 \quad$ Figure 6.
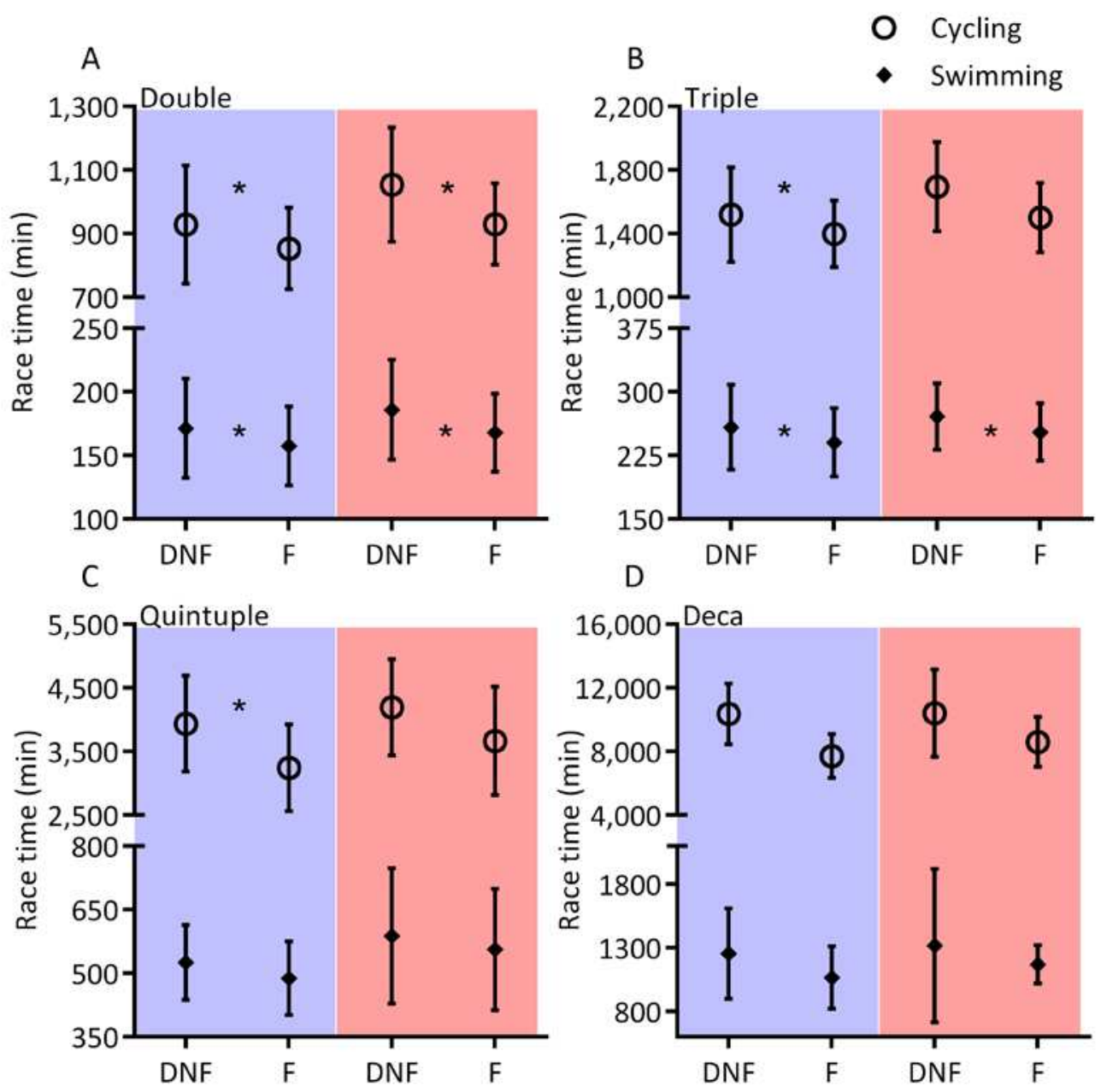

423 